Referencia para citar este artículo: Ancheta-Arrabal, A. (2019). Equidad y educación de la primera infancia en la agenda educativa mundial. Revista Latinoamericana de Ciencias Sociales, Niñez y Juventud, 17(1), 47-59. doi:https://dx.doi.org/10.11600/1692715x.17102

\title{
Equidad y educación de la primera infancia en la agenda educativa mundial ${ }^{*}$
}

\author{
ANA ANCHETA-ARRABAL ** \\ Profesora Universidad de Valencia, España.
}

\begin{abstract}
Artículo recibido en septiembre 11 de 2017; artículo aceptado en noviembre 2 de 2017 (Eds.)
\end{abstract}
- Resumen (descriptivo): el presente trabajo se inspira en la necesidad de abordar un análisis de la equidad en la etapa de educación infantil desde un enfoque holístico, para contribuir al debate actual sobre la importancia de la equidad en la nueva agenda educativa y sus metas para la primera infancia. Parte de fundamentos del estudio teórico de la equidad en educación desde un enfoque holístico que permita aplicar este análisis a la educación de la primera infancia en las sociedades iberoamericanas, en aras de aportar una perspectiva más amplia en la discusión de sus logros y desafios. Finalmente, este entendimiento posibilita la interpretación de los límites y posibilidades de los objetivos globales establecidos para la educación de la primera infancia y la medición de los progresos e implicaciones para la equidad educativa.

Palabras clave: educación de la primera infancia, equidad, agenda mundial educativa, indicadores (Thesaurus for Education Systems in Europe).

\section{Equality and early childhood education in the global education agenda}

- Abstract (descriptive): The present paper is inspired by the need to carry out an analysis of equality in early childhood education using a holistic approach and contribute to the current debate regarding the importance of equality in the new education agenda and its goals for early childhood. This analysis is based on the foundations of the theoretical study of educational equality from a holistic perspective, which facilitates its application to early childhood education in Ibero-American societies. This will provide a broader perspective on the discussion of the achievements and challenges in the area of equality. Finally, the understandings reached as a result of this analysis facilitate the interpretation of the limits and possibilities of global education targets for early childhood, the measurement of progresses and implications for educational equality.

Key words: Early childhood education, equality, global education agenda, indicators (Thesaurus for Education systems in Europe).

\section{Equidade e educação na primeira infância na agenda mundial de educação.}

- Resumo (descritivo): o presente trabalho tem como inspiração analisar a equidade na educação infantil a partir de um enfoque holístico. Busca contribuir para o debate sobre a importância da equidade na nova agenda educativa tendo em vista as metas a serem alcançadas

Este artículo de revisión se basa en la investigación «Equidad y educación de la primera infancia en la agenda educativa mundial» realizada por la autora con la financiación de la Universidad de Valencia. (Contrato I8F0VFPDYMAF1240 del 04/06/2014 hasta la fecha). Gran área: Ciencias de la Educación. Área: Educación General. Subárea: Educación Infantil.

** Maestra y Pedagoga Infantil. Doctora en Educación bajo el programa de Estudios Sociales y Políticos de la Educación de la Universidad de Valencia (UV) con Mención Europea/Internacional. Profesora Contratada Doctora del Departamento de Educación Comparada e Historia de la Educación de la UV. Orcid: 0000-0002-9833-242X. Índice H5: 7. Correo electrónico: ana.ancheta@uv.es 
para a educação na primera infância. Apresenta os fundamentos da equidade a partir de um estudo teórico com enfoque holístico e, desde aí, amplia-se para a análise da equidade em educação, mais especificamente na Educaçao Infantil das sociedades iberoamericanas, no intuito de aportar uma perspectiva mais ampla na discussão de suas conquistas e desafios. Conclusivamente, este estudo ajuda para a interpretação dos limites e possibilidades dos objetivos globais estabelecidos para a educação na primeira infância e para a mediação dos progressos e implicações considerando o alcance de metas de equidade educativa.

Palavras-chave: educação na primeira infancia, equidade, agenda mundial educacional, indicadores (Thesaurus for Education Systems in Europe).

-1. Introducción. -2. Equidad en educación y justicia educativa desde un enfoque holístico. -3. Educación de la primera infancia y equidad. -4. Indicadores para medir la equidad educativa en la Agenda de Objetivos de Desarrollo Sostenible: ¿en la búsqueda de la justicia educativa desde la base? -Lista de referencias.

\section{Introducción}

Durante las últimas décadas los países iberoamericanos han abordado grandes retos educativos, como la alfabetización y escolarización obligatoria, a la falta de solucionar como asignatura pendiente la equidad de los sistemas educativos; esta última constituye actualmente uno de los mayores desafíos para el progreso social en el mundo. De este modo, las oportunidades educativas de las familias y los niños y niñas que más se pueden beneficiar de la educación se ven reducidas, perpetuando los patrones de desventaja y exclusión social (Marshall, 1961). A tales desigualdades educativas derivadas del nivel de renta o la condición social (por las necesidades especiales o el lugar de residencia de las personas) se han sumado otras originadas por el lugar de procedencia debido a los procesos migratorios desencadenados por el fenómeno de la globalización. Efectivamente, en la base del problema señalado «es posible que se formulen y aprueben políticas educacionales teniendo en cuenta los derechos humanos, pero muy frecuentemente el acceso a la escuela y a una buena educación están determinados por consideraciones del mercado y las prevalecientes desigualdades sociales, económicas, culturales y geográficas (incompatibles con las convenciones sobre derechos humanos)» (Hallak, 2001, p. 16). En un contexto de inequidad en el ejercicio del derecho a la educación, el mayor nivel educativo contribuye al mejoramiento de la calidad de vida de las personas, pero al mismo tiempo profundiza los problemas de desigualdad existentes en la región iberoamericana, registrándose pocos avances al respecto (Organización de Estados Iberoamericanos, 2010, p. 54). En la primera infancia esta falta de equidad como punto de partida acentúa las desigualdades derivadas de la condición económica y social (Burger, 2013) y, en el largo plazo, supone grandes diferencias en el desarrollo vital y para el riesgo de exclusión social de los grupos más desfavorecidos (Pérez-Escamilla, Rizzoli-Córdoba, Alonso-Cuevas, \& Reyes-Morales, 2017).

En este momento, la democratización de la educación, en general, y el papel que la educación de la primera infancia juega en la igualdad de oportunidades educativas de las personas, en particular, se han convertido en premisas para la equidad y la movilidad social en el escenario global (Unesco, Unicef, \& World Bank, 2015). Así, la educación en la primera infancia se ha establecido como estrategia universal que puede producir un alto beneficio tanto individual como social, fundamentada en que su principal ganancia está dirigida - por definición - a hogares social, cultural y económicamente desaventajados (Young, 1996) y en que es una fase decisiva para el ciclo de la vida del ser humano cuya atención integral permite enfrentar los retos de la pobreza, la inequidad y la exclusión social (Rosemberg, 1999).

En coherencia con el conocimiento desarrollado sobre las potencialidades y los beneficios de la educación de la primera infancia, esta etapa ocupa una de las posiciones estratégicas de las actuales agendas de desarrollo y educación en el reconocimiento de su contribución para la mejora y consecución 
de sus objetivos y metas, así como para su elevación en la escala de prioridades políticas (Unesco, 2010). Sin embargo, las diferencias tanto en el acceso como en la duración de la escolarización en esta etapa educativa indican que la población infantil accede a la educación obligatoria con unos niveles de preparación distintos, de modo que la plataforma de aprendizaje que supone la misma puede ser menos sólida para unos grupos de infantes que para otros, especialmente en las edades más tempranas (Organización para la Cooperación y el Desarrollo Económico, 2013).

A estas disparidades se añaden ahora las fuertes brechas sociales fruto de la digitalización de unas sociedades contextualizadas, a su vez, en el escenario global de inestabilidad y crisis económica (Vargas-Barón \& Schipper, 2012).

Cierto es que cada crisis está influenciada por su propia complejidad cultural, pero tendencias actuales (como la desigualdad) representan un nuevo capítulo en el estudio del impacto de estos fenómenos para el desarrollo de sistemas socialmente equitativos de educación para y en la primera infancia. En este sentido, más allá de sus tradicionales funciones propedéutica y preventiva, la educación de la primera infancia se sitúa en un lugar central por el carácter ecualizador otorgado en la lucha para garantizar la equidad determinante en el desarrollo humano. Así, en los países iberoamericanos queda abierta toda la discusión sobre las políticas compensatorias y la significativa importancia de esta etapa educativa (Tedesco, 2009, p. 86).

Ahora bien, resolver la cuestión de lo que cuenta como equidad en la educación de la primera infancia debe constituir un debate obligado en la construcción de este discurso; ejercicio este que no es posible hacer desde niveles puramente abstractos, en la medida que solo puede ser entendida correctamente en sus contextos de interpretación y representación. Es decir, el estudio de las desigualdades actuales ha de ir más allá de la atención global desde el discurso de los derechos de la primera infancia al análisis de las prioridades locales donde las influencias de poder desde diferentes sectores (económicos, sociales, ideológicos...) determinan las vidas de los más pequeños dentro y entre países.

A tales efectos, la finalidad fundamental de este trabajo es abordar un marco analítico de la equidad en la primera infancia que permita atender a la realidad actual de los países en lo que respecta al bienestar social y la exclusión social como fuerzas que condicionan todos los procesos de desarrollo para, de este modo, poder entender la situación de las iniciativas educativas para la primera infancia, en especial para los grupos más vulnerables. El objetivo no es otro más que el de tratar de determinar qué cuenta —o debería contar- como equidad en el marco de las interpretaciones actuales para la educación de los más pequeños en sociedades locales, y si ello limita o expande las medidas actuales cuando se eleva esta cuestión al nivel de las decisiones políticas internacionales y del establecimiento de su seguimiento. Habida cuenta de que lograr tal empeño, empero, exige de un espacio de dimensiones superiores de las que se dispone, se pretende abrir la posibilidad a dicha discusión como cuestión que, cuanto menos, debería ser un área de atención institucional urgente por su impacto en la realidad local y la responsabilidad global, en la paradoja de un mundo que se torna cada vez más desigual, al tiempo que acuerdos globales en la búsqueda de la mejora son firmados por los líderes mundiales.

\section{Equidad en educación y justicia educativa desde un enfoque holístico}

La equidad en educación es, en general, una cuestión asociada a la justicia social (Vincent, 2003) y es actualmente foco de atención de las agendas globales, así como un tema de discusión para los diversos actores implicados en los diferentes contextos educativos. De tal manera, el uso del término equidad en el discurso educativo por parte de políticos, investigadores y profesionales está en sí mismo fuertemente influenciado por los escenarios donde las discusiones sobre equidad se sitúan y desde donde deben ser analizados. En palabras de Hutmacher (2001), en educación la equidad es fruto del proceso de toma de decisiones de las autoridades educativas, al considerar las diversas posibilidades, ventajas o desventajas y las oportunidades reales del alumnado de un nivel educativo, 
en aras de proporcionar una justa distribución o atribución y adquisición de recursos.

De este modo, la equidad entra en escena cuando la justicia, entendida aquí como igualdad de oportunidades educativas, no debería reproducir o incrementar las oportunidades o desigualdades sociales. Pero, además, tal como Bronfenbrenner (1973) ya apuntara, la equidad (equity) referida a la justicia social o a la justicia (fairness) implica una moral subjetiva o un juicio moral. Es decir, los juicios individuales o colectivos a propósito de la equidad o justicia de un determinado nivel de igualdad observado pueden diferir puesto que la equidad implica juicios de valor y distintos modos de entender según los diversos sistemas de valores de las sociedades y de los individuos.

Esta cuestión central es también abordada por Sen (1992) como un problema de definición, no del concepto de equidad en sí mismo, sino de su objeto cuando se convierte en la medición de las desigualdades a través de indicadores cuya relevancia, a su entender, puede ser legítimamente cuestionada por defensores de otro principio de justicia. Con todo, el debate sobre el significado preciso de la equidad como concepto normativo persiste a través del tiempo, en la medida que sus diversas interpretaciones subyacen a su propio carácter subjetivo según se concreta.

En términos de equidad educativa, la amplia mayoría de políticas públicas han venido resolviendo su discusión sobre la base de clasificaciones e indicadores estadísticos que teóricamente establecen la consecución o el grado de alcance de la misma. De aquí la necesidad de superar la medición y evaluación de la equidad educativa desde una metodología cuantitativa a una cualitativa desde un enfoque holístico.

Los enfoques educativos de la equidad desde dicho enfoque holístico de su investigación han sido clasificados en tres (Moreno-Herrera, 2005):

a) La equidad desde su contextualización, donde la investigación sobre la equidad en educación centra su foco de análisis en la contextualización de las prácticas y escenarios de representación de la misma.

b) La equidad desde la perspectiva de la teoría curricular y el análisis discursivo, en relación al desplazamiento performativo de la equidad como concepto estudiado.

c) La equidad analizada desde la perspectiva de los indicadores, donde los argumentos de esta aproximación descansan en la lógica de que las inequidades educativas dependen de las desigualdades en la sociedad, en general, y de las desigualdades de los sistemas educativos, en particular.

Desde este último enfoque, las cuestiones de equidad en la provisión de la educación se tornan progresivamente políticas en esencia y se convierten en un centro de interés y discusión para los actores implicados: políticos, académicos y ciudadanos. En cuanto a este hecho en la educación de la primera infancia, la equidad se enfrenta con los modelos actuales en los que su provisión llega a la población a la que se destina, si bien ello afecta a los niveles de justicia social de toda la población en conjunto en las diversas sociedades, según el paradigma actual de la educación en el mundo.

En la base de esta perspectiva amplia y holística se considera que el modo en que las políticas, los servicios, las familias y las comunidades pueden apoyar la educación primera infancia es determinante y su análisis resulta fundamental para las discusiones de las diversas opciones políticas que los responsables en la toma de decisiones pueden tener en cuenta en aras del promover la equidad en la educación de los niños y niñas más pequeños. Es decir, se trata de abordar la equidad en esta etapa educativa desde su contexto, para lo cual es preciso conocer si las circunstancias de la primera infancia son tales como para que niños y niñas sean capaces de convertirse en lo que pueden llegar a ser, o en qué sentido son desaventajados como los modos que imposibilitan su plena participación en la vida y las oportunidades que su mundo alrededor les ofrece (Umayahara, 2004). En consecuencia, no se trata tanto de pensar en la función propedéutica de esta etapa vital como factor clave para cambiar las inercias sociales y modos en los que opera la desigualdad, sino más bien de repensar los modos para garantizar equitativamente a todos los niños y niñas pequeños las posibilidades de aprendizaje para mejorar, tanto su actual situación y desarrollo, como su integración en la sociedad a 
pesar de las dinámicas y rápidas transformaciones de esta (Kernan, 2012).

Todo ello pone de manifiesto que la equidad en esta etapa debe constituir en sí misma un fin desde las edades más tempranas, de manera que se haga efectivo el bienestar infantil en el presente y no solo un tipo de medio para posibilitar una deseable y futurible justicia social a la que, en todo caso, está supeditada. Así, adoptar un enfoque en pro de la equidad en las políticas y programas de educación de la primera infancia, supone crear «un campo de juego más igualitario al garantizar que todos los niños y niñas tengan oportunidades de recibir primeras experiencias, reduciendo la desigualdad educativa de resultados» (Grupo Consultivo de Atención y Desarrollo de la Primera Infancia, 2016, p. 16).

La atención institucional efectiva a este primer nivel educativo resulta, pues, fundamental para posibilitar el ejercicio de los derechos humanos que asegure una educación de calidad inclusiva que ha de empezar en esta etapa (Blanco, 2005). Con el fin de hacer efectivo el principio de equidad en el ejercicio de este derecho, las administraciones públicas desarrollan acciones de carácter compensatorio en relación con las personas, grupos y ámbitos territoriales que se encuentren en situaciones desfavorables, al tiempo que proveen los recursos económicos y apoyos precisos para evitar desigualdades derivadas de factores sociales, económicos, culturales, geográficos, étnicos o de cualquier otra índole.

La equidad en el acceso a la educación infantil puede ser actualmente una realidad que se tiene muy en cuenta como prioridad para determinados grupos etarios de población infantil, pero también se relaciona con la desigualdad de género que los programas educativos - incluso para estas edadestipifican, en los aspectos del contenido, de la organización del aula, en la estructura del centro de educación y atención de la primera infancia, en el comportamiento del educador hacia este grupo de edad en función del género y en el ambiente general (Chartier \& Geneix, 2006). En este terreno, como en el resto de políticas educativas, equidad y calidad integran dos aspectos diferenciados del mismo hecho, la educación, en aras de garantizar la equidad educativa como un elemento propio de la mejora, y no solo un compendio de medidas especiales (Demeuse, Crahay, \& Monseur, 2001). De hecho, un acceso equitativo es el primer paso para promover la equidad en la educación de la primera infancia; pero, a su vez, es necesario examinar la equidad de su distribución y la calidad de su provisión, como un prerrequisito para poder garantizar la equidad. Aludiendo al segundo referente mencionado en el enfoque holístico para el estudio de la equidad educativa, como objeto estudiado en relación con la teoría curricular, es necesario profundizar desde la perspectiva del análisis discursivo en la performatividad de dichas acciones para la primera infancia y la justicia social.

A este respecto, el discurso se basa en las evidencias derivadas de la investigación sobre los beneficios de las políticas o programas educativos para los niños y niñas más pequeños, en especial para los más desfavorecidos, desde la consideración de que objetivos mundiales como el de reducir la pobreza global, están supeditados a alcanzar la equidad en aspectos tan determinantes como la salud o la educación de las futuras generaciones (Barnett, 1997). Ahora bien, en este tipo de políticas que consisten en invertir en los grupos más vulnerables, «es raro que una iniciativa de política pública que promueve la equidad y la justicia social, promueva al mismo tiempo la productividad en la economía y la sociedad en su conjunto» (Heckman, 2006, p. 2). La certeza de esta relación, desde la perspectiva del desarrollo humano, aunque debatida es innegable, pues la correlación entre la igualdad de oportunidades y el bienestar en la primera infancia y su impacto en la educación, la salud, el capital social de las sociedades, y la equidad en su conjunto, resulta fundamental para un crecimiento económico y social en clave de sostenibilidad (Sen, 2004). Así, resulta de vital importancia entender cómo y hasta dónde la sociedad provee los servicios de educación para la primera infancia y si de verdad esta es valorada; pero, además, el alcance de su impacto ha llevado al reconocimiento de que ha de tratarse como una cuestión de las políticas públicas integrada en distintas áreas que ha de recibir la inversión pública y atención institucional apropiadas (Britto \& Sherr, 2016).

Con todo, obtener información completa y detallada para atender a los grupos en situaciones de riesgo o vulnerabilidad exige una mayor colaboración intersectorial, tanto entre diferentes instancias gubernamentales como entre estas e instituciones no gubernamentales, así como efectuar cambios 
estructurales y de contenido para otorgar a la primera infancia la prioridad que se merece. De este modo, para hacer posible y promover el desarrollo de políticas y programas que atiendan de forma prioritaria a las poblaciones más vulnerables resulta imprescindible analizar críticamente la situación mediante información cuantitativa y cualitativa acerca del contexto demográfico, social y económico de la población de la primera infancia, así como las características de su educación (Ancheta-Arrabal, 2013). En esta tarea resulta igualmente determinante reconocer la importancia de esta labor en la contribución a una educación de la primera infancia en la superación de la exclusión (Blanco \& Umayahara, 2004). Por ello, se considera fundamental abordar un tratamiento sistemático de la situación de la educación de la primera infancia y la profundización del análisis de las relaciones entre las diferentes factores o variables, con el fin de identificar aquellos que están afectando a la participación en esta etapa educativa (Sistema de Información de Tendencias Educativas en América Latina-Siteal, 2008).

Desde el enfoque holístico de la equidad en la educación que sirve aquí de referente, se corresponde con el análisis de los indicadores que miden las desigualdades educativas como herramienta importante para valorar, en este caso, cómo se efectiviza la atención institucional hacia la educación de la primera infancia en las políticas públicas y valorar sus implicaciones en el desarrollo de los más pequeños. En esta línea, el análisis de la equidad educativa a través del desarrollo de indicadores ya constituye, de manera general, un marco de referencia y actualidad en la comunidad internacional para el seguimiento de las agendas educativas como la nueva Agenda de Desarrollo Sostenible para 2030 y, en particular, en lo referente a la educación de la primera infancia (Open Working Group, 2014). Para el interés de este trabajo, esta perspectiva de los indicadores para analizar la equidad en la educación primera infancia y su desarrollo en el contexto iberoamericano es el foco de atención que abre la discusión.

\section{Educación de la primera infancia y equidad}

Como se acaba de exponer, la equidad en la agenda de la educación para la primera infancia constituye una inquietud recurrente que se ha establecido como una prioridad de la política global. De hecho, la actual Agenda 2030 para el Desarrollo Sostenible va más allá de la atención y la educación de los más pequeños como medio para satisfacer sus necesidades de aprendizaje e incluye el desarrollo de la primera infancia como un resultado (Unesco, 2017, p. 242).

En Iberoamérica los gobiernos se comprometieron en la X Cumbre Iberoamericana de Panamá en el año 2000 a «continuar impulsando políticas y programas nacionales que promuevan el desarrollo con equidad y justicia social, procurando asignar mayores recursos al gasto social, en especial en salud, educación, cultura y ciencia y tecnología»; así como a realizar esfuerzos para garantizar el «acceso a una educación inicial temprana, y a la educación primaria gratuita y obligatoria, sustentada en los principios de no discriminación, equidad, pertinencia, calidad y eficacia» (Organización de Estados Iberoamericanos, 2000). La característica más destacable de esta concepción de la educación en la primera infancia es el papel cada vez más importante que ha adquirido en las últimas décadas como segmento del sistema educativo en la compensación de desigualdades, identificándola como otro factor estratégico para garantizar la equidad, entendida como igualdad de oportunidades de acceso, permanencia y logros educativos (Organización de Estados Iberoamericanos, 2015). De este modo, las autoridades educativas iberoamericanas han reconocido la educación de la primera infancia no solo como un derecho de todos los niños y niñas, sino también como una oportunidad.

En este sentido, los países iberoamericanos han realizado esfuerzos significativos durante las últimas décadas para aumentar la expansión de la educación de la primera infancia, especialmente allí donde este reto era mayor y, a su vez, favoreciendo los niveles de cobertura de la educación primaria (López, 2009, p. 37). Sin embargo, tal y como se señaló anteriormente, abarcar cuestiones como la equidad y la calidad en la provisión de la educación y, concretamente, en la etapa que nos ocupa, implica su estudio desde un enfoque holístico, en aras de poder determinar las causas de desigualdad 
social y poder tomar medidas preventivas desde los primeros años (Smidt, 2010). A este respecto, cabe recordar que el desarrollo histórico de este nivel muestra como rasgo característico la dualidad, tanto en lo que respecta a la diversificación inicial de la oferta, como en lo que afecta a la distribución de los servicios, resultando poco equitativa atendiendo a términos de cobertura y contenido como para propiciar una verdadera democratización educación de la primera infancia de calidad para todos (Mialaret, 1976).

En cuanto a la diversificación de la oferta, el elenco de su provisión abarcaba desde unos espacios de experimentación y especificidad pedagógica, hasta otros que se definían por una fuerte impronta de los «cuidados de tipo médico-sanitarios frente a la preocupación por la situación de abandono infantil ante la creciente incorporación de la mujer al mercado de trabajo y la extrema pobreza en que vivían un número importante de familias» (Harf et al., 1996, p. 19, citado por Dicker, 2002). Ante esta falta de equidad constatada, particularmente con respecto a las edades más tempranas, surgieron los programas no formales como acciones integradas entre los diversos sectores sociales para mejorar las condiciones de vida de los más pequeños, a partir del desarrollo global y progresivo de sus propias comunidades mediante un esfuerzo pedagógico destacable que intentaría hacer una síntesis de las dos pedagogías emergentes anteriormente mencionadas (Young \& Fujimoto, 2003).

El posterior reconocimiento - tanto de los responsables políticos como de los expertos- de que un acceso equitativo a una educación y cuidado de calidad durante esta etapa puede reforzar las bases del aprendizaje a lo largo de toda la vida, daría lugar a la adopción de distintos tipos de medidas en los países iberoamericanos para la provisión de una respuesta de sus sistemas educativos al reto que planteaba la institucionalización y provisión de esta etapa educativa como factor integrante de las políticas educativas para garantizar el derecho a la educación.

En general, parece que los países con una mayor tradición histórica en la provisión de esta etapa y mayor atención institucional a la primera infancia son aquellos que han desarrollado mayores acciones legislativas y políticas para los grupos vulnerables; por su parte, en el resto permanecen pendientes las tareas de sensibilización, desarrollo de políticas efectivas de integración, así como de la recopilación de la información y estadísticas pertinentes en este terreno (Unesco, 2004, p. 19). La problemática de las necesidades de cobertura, unida a la falta de recursos asignados desde la administración pública para esta etapa educativa, conllevó una falta de equidad en la atención educativa a los niños y niñas de estas edades, privilegiando aquellos que con mejores condiciones para recibir este tipo de enseñanza, como son los grupos que viven en zonas urbanas altamente pobladas. Así, se relegó a un segundo plano a quienes su atención resultaba más difícil o compleja, como aquellos de zonas no urbanas o campesinas, de localidades marginadas o de minorías étnicas regionales (Unesco, 2007). Por tanto, es un hecho constatado que el primer indicio de desigualdad se registraba ya en el acceso a la educación en los primeros años de edad, constituyendo una de las áreas más problemáticas en relación con la equidad, que caracteriza la dinámica de los sistemas educativos en sus distintos niveles y áreas e implica que los grupos y clases más desfavorecidos tengan resultados académicos más bajos, menos oportunidades de acceso a la educación y permanezcan menos tiempo en el sistema educativo (Comisión Económica para América Latina y el Caribe, 2010). Así, la consecución de acuerdos internacionales en los derechos de la infancia se ha visto mermada por la falta de su impacto en las políticas públicas pertinentes (Ramírez, 2011).

En la práctica los avances para favorecer a los grupos sociales en desventaja han sido limitados, pues «por comparación con los grupos más favorecidos, su situación no ha mejorado de forma significativa» (Oficina Regional de Educación para América Latina y el Caribe, 2013, p. 4). Con todo, el moderno discurso de la equidad en la educación de la primera infancia no ha apuntado realmente a asegurar iguales oportunidades de aprendizaje para todos, pues no puede asegurarse exclusivamente desde la igualdad de acceso a la educación, sino desde intervenciones integrales y estructurales de orden político, económico y social; de ahí la necesidad de entender la educación de la primera infancia no como una política sectorial, sino como parte de otras políticas como la social y la económica.

Reconociendo la inequidad como gran tarea pendiente y sus consecuencias para el desarrollo 
de los países, la Agenda Educativa Post-2015 ha establecido como principio rector la reducción de la desigualdad y la pobreza a través de la provisión de una educación de calidad para todos, la cual contribuya en el avance hacia unas sociedades más inclusivas (Unesco, 2013). Igualmente, en dicha reducción de la inequidad y la pobreza a través de la provisión de una educación inclusiva de calidad, el impacto de la educación se revela como esencial para lograr un desarrollo humano sostenible, sobre la base del aprendizaje a lo largo de la vida y el valor de la riqueza de la diversidad cultural (Unesco, 2014). De este modo, para salvar la distancia de la inequidad educativa, la garantía política para convertir las aspiraciones en realidad a través de procesos de acciones concretas como el desarrollo de esta agenda y de indicadores para valorar eficazmente la equidad se convierte en un requisito previo para trabajar por la justicia social, tal y como se aborda seguidamente.

\section{Indicadores para medir la equidad educativa en la Agenda de Objetivos de Desarrollo Sostenible: ¿en la búsqueda de la justicia educativa desde la base?}

El nuevo objetivo mundial para la educación en la agenda de Objetivos para el Desarrollo Sostenible (ODS) para 2030 de «garantizar una educación inclusiva, equitativa y de calidad y promover oportunidades de aprendizaje durante toda la vida para todos» requiere, según el Grupo Asesor Técnico ${ }^{1}$ (TAG, por sus siglas en inglés) de la Unesco, que en la actual agenda educativa la comunidad internacional aborde dos desafíos cruciales (Grupo Asesor Técnico, 2015), a saber: 1) la medición de los resultados de aprendizaje; y 2) el mejoramiento de las mediciones de equidad en la educación. En este sentido, en el documento de referencia Indicadores temáticos para el monitoreo de la Agenda educativa 2030 (elaborado por el TAG para debatir sobre indicadores) se pone de manifiesto la necesidad de desagregar los datos en referencia a los indicadores propuestos para medir la meta para la educación de la primera infancia, así como el uso consistente de los indicadores propuestos para la recopilación efectiva y significativa de información. De modo explícito, en referencia a la meta 4.2 («Para 2030, velar por que todas las niñas y todos los niños tengan acceso a servicios de calidad en materia de atención y desarrollo en la primera infancia y enseñanza preescolar, a fin de que estén preparados para la enseñanza primaria») se expresaba la necesidad de seguir trabajando con el fin de examinar alternativas al índice de Desarrollo de la Primera Infancia, incluido en la encuesta MICS ${ }^{2}$, para medir la preparación para la educación obligatoria de manera consensuada y en coherencia con la necesidad de estudiar la equidad transversal entre las metas.

De hecho, entre las dificultades señaladas que plantea el seguimiento en relación a esta meta, en cuanto al acceso y la participación, se destaca que no hay ninguna suficiente información sobre el número de niños y niñas que se benefician de la educación infantil durante al menos un año, para lo que se proponen dos indicadores complementarios relacionados con la participación extraídos de datos administrativos a partir de informes escolares y de encuestas a los hogares (Unesco, 2017, p. 242). Asimismo, la propuesta incorpora una recomendación para ampliar el indicador que mide si la primera infancia se encuentra bien encaminada en términos de desarrollo e incluya la edad de participación en programas organizados de aprendizaje (Grupo Asesor Técnico, 2015, p. 10).

Desde esta mirada sistemática a la dimensión potencial de la desventaja educativa, se requiere contar con datos desagregados sobre las personas derivados de diversas fuentes (entre ellas, hogares, encuestas escolares y fuentes administrativas), para lo que esta propuesta de indicadores del TAG permite hacer un seguimiento del avance logrado en términos de reducir las desigualdades en todas las áreas de interés identificadas para las metas de educación (Open Working Group, 2014).

1 El Grupo Asesor Técnico fue creado por la Unesco para facilitar retroalimentación respecto de las metas de educación propuestas para el Post-2015, elaborar recomendaciones sobre los indicadores y establecer una agenda de mediciones para mantener informado y proporcionar asistencia técnica al Comité de Dirección de la EPT. Presidido por el Instituto de Estadística de la Unesco, el TAG lo integran expertos del Informe de Seguimiento de la EPT en el Mundo, la OCDE, Unesco, Unicef y el Banco Mundial.

2 La evidencia de la importancia del desarrollo de la primera infancia ha dado lugar a estas mediciones basadas en la investigación, siendo una de ellas recabada a través de la encuesta MICS de la Unicef, aunque en la práctica no existe consenso en un indicador que pueda ser recabado en forma costo-eficiente y comparado entre diversos países, particularmente entre los de ingresos bajos y altos (Grupo Asesor Técnico, 2015). 
Adicionalmente, para facilitar la comparación, se recomienda la coordinación de las encuestas en cuanto a las preguntas relativas a la primera infancia y la enseñanza preescolar, de modo que puedan calcularse unas estadísticas mejoradas para supervisar las tasas de participación comprendidas en la meta 4.2; así como hacer acopio de información más detallada sobre los programas y proveedores de enseñanza preescolar para mejorar la medición de las posibilidades estructuradas de aprendizaje (Unesco, 2017, p. 247). Finalmente, se recuerda que la agenda considera necesario adoptar un enfoque explícito en cuanto a la equidad, que se incluye en metas específicas tales como la meta 5 (equidad entre los géneros) y la meta 10 (reducción de las desigualdades), pero que, a su vez, los indicadores de educación deben intentar captar también las variaciones entre los diferentes sub-grupos de la población definidos sobre la base de características grupales o individuales, es decir, sexo, riqueza, área geográfica, etnicidad, idioma o discapacidad (o sus combinaciones) (Grupo Asesor TécnicoTAG, 2015). En este sentido, la equidad en la educación resulta más difícil de capturar en su sentido más amplio, pues estas metas se limitan a medir la disparidad (Unesco, 2017, p. 256). De acuerdo con el Grupo Consultivo de Atención y Desarrollo de la Primera Infancia (2016), el marcador de la equidad para la nueva agenda de la primera infancia «es el grado al cual contribuye a lograr resultados iguales en educación, empleo y otros aspectos del bienestar» (p. 25).

En cualquier caso, cabe enfatizar aquí la importancia de indagar en mayor profundidad en algunos aspectos en lo que concierne a la equidad en la educación de la primera infancia, en aras de establecer una investigación más rigurosa y exhaustiva que refleje la realidad a través del diseño de indicadores desde un enfoque holístico de esta meta. Para tal fin resulta pertinente la interpretación referente a los indicadores y verificadores del cumplimiento del derecho a la educación que planteara la primera Relatora del Derecho a la Educación (1998-2004), Katarina Tomasevski, desde sus «cuatro as»: asequibilidad, accesibilidad, aceptabilidad, adaptabilidad (Tomasevski, 2006). A pesar de que las características propuestas desde este enfoque están referidas al sistema de educación gratuita y obligatoria, resultan referentes igualmente útiles en el intento de medir la democratización el derecho durante al menos un año de educación en la primera infancia (señalado por la Unesco y que garantiza la mayoría de países iberoamericanos) como, dada su interdependencia, a una educación de calidad. Igualmente, se vuelve imprescindible abordar los problemas de cohesión interna que estas acciones entrañan, descubriendo las dificultades y el reto que todos los sistemas educativos deben asumir para facilitar el proceso de transición que los niños y niñas afrontan al entrar en educación primaria y la multiplicidad de opciones políticas en las que han derivado las posiciones adoptadas frente a este desafío (Woodhead \& Moss, 2007). Este aspecto, lleva necesariamente a la reflexión de otro de los tópicos de la equidad alrededor del acceso universal y apropiado a la educación de la primera infancia que todos los niños y niñas deberían disfrutar como derecho propio de países que han ratificado la Declaración de los Derechos Humanos y la Convención de los Derechos del Niño (1989), base valiosa en la que se debe interpretar la aportación documental (Kochen, 2013).

La discusión del derecho a la educación en la primera infancia desde una interpretación de la equidad reitera tres puntos (Myers, 2009):

1. Cuando hablamos del derecho de la educación debemos hablar del derecho de la educación de calidad aplicada con equidad.

2. Decir que la educación debe ser para todos no es decir que la educación para todos debe ser lo mismo en cada lugar.

3. Los documentos que marcan la educación como un derecho también empiezan a dibujar características de la educación a que se tiene derecho, características que vale la pena tomar en cuenta para formular ideas sobre la calidad y equidad de la educación.

Se trata, en definitiva, de concebir una equidad de todos los seres humanos desde su etapa inicial de vida que, al mismo tiempo, deje espacio suficiente para el reconocimiento de la diversidad de capacidades adquiridas o potenciales, en la medida que la igualdad de oportunidades implica equidad de posibilidades de revelar diferencias. Ello implica pasar de la concepción de una infancia de las necesidades a una infancia de los derechos (De los Ángeles-Bautista, 2001), superando la 
visión utilitarista y economicista que anula a los sujetos centrales - los niños y niñas - e impide su participación activa, alimentando grandes asimetrías estructurales, además de reducir la riqueza cultural y la diversidad intrínseca de los procesos pedagógicos (Muñoz, 2013). Asimismo, cabe llamar a una ética de la calidad y equidad de los servicios para la primera infancia, hechos que dependen, entre otros aspectos, de la implicación y atención institucional de los actores que intervienen en esta etapa (Blanco, 2008).

A tales efectos, la investigación sobre la primera infancia tiene mucho que aportar al desarrollo de las políticas y prácticas de sus derechos, así como la supervisión y evaluación lo hace sobre su impacto para superar las limitaciones de la investigación tradicional, en el cometido de dar cuenta de la mercantilización y distorsiones que ello puede producir en la equidad (Sosinsky, Lord, \& Zigler, 2007). Igualmente, conviene no olvidar que ambas derivan de las tradiciones europea y norteamericana (Penn, 2008), como una particular forma de diferenciación adquirida en el modo que se concibe la primera infancia desde los distintos puntos de vista culturales, sociales y particulares (Cook, 2004, p. 41). Por todo ello, resulta de vital importancia situar los derechos y la equidad de la primera infancia en cada contexto cultural y socioeconómico para el desarrollo humano (Montgomery, Burr, \& Woodhead, 2003) y no aplicar con carácter universal patrones occidentales que dificulten los objetivos para esta etapa vital.

\section{Lista de referencias}

Ancheta-Arrabal, A. (2013). El desarrollo y seguimiento de las políticas para educación y atención de la primera infancia en el contexto europeo. Perfiles Educativos, 35(140), 134-148. doi: https:// doi.org/10.1016/S0185-2698(13)71826-9

Barnett, W. S. (1997). Costs and financing of early child development programs. En M. E. Young (Ed.) Early child development. Investing in our children's future. Amsterdam: Elsevier Science.

Blanco, R. (2005). La educación de calidad para todos empieza en la primera infancia. Enfoques Educacionales, 7(1), 14-18.

Blanco, R. (2008). Construyendo las bases de la inclusión y la calidad de la educación de la primera infancia. Revista de Educación, 347, 33-54.

Blanco, R., \& Umayahara, M. (2004). Síntesis regional de indicadores de la primera infancia. Santiago de Chile: Orealc, Unesco.

Britto, P. R., \& Sherr, L. (2016). A road less travelled: Early childhood evidence to investment. Vulnerable Children and Youth Studies, 11(3), 193-210, doi:10.1080/17450128.2016.1220659

Bronfenbrenner, M. (1973). Equality and equity. The ANNALS of the American Academy of Political and Social Science, 409(1), 9-23.

Burger, K. (2013). Early childhood care and education and equality of opportunity: Theoretical and empirical perspectives on social challenges. Springer: Wiesbaden.

Comisión Económica para América Latina y el Caribe-Cepal. (2010). Pobreza infantil en América Latina y el Caribe. Santiago de Chile: Cepal, Unicef.

Cook, C. P. (2004). Cross-cultural perspectives on the child image. En A. Weyts (Ed.) Ghent on children's rights, (pp. 35-47). Ghent: University of Ghent.

Chartier, A. M., \& Geneix, N. (Eds.) (2006). Petite enfance: enjeux éducatifs de 0 à 6 ans. Paris: Issy les Moulineaux.

De los Ángeles-Bautista, F. (2001). Rights from the start: ECD and the Convention on the Rights of the Child (CRC). Early Childhood Matters, 98, 8-22.

Demeuse, M., Crahay, M., \& Monseur, Ch. (2001). Efficiency and equity. En W. Hutmacher, D. Cochrane, \& N. Bottani (eds.) In Pursuit of equity in education. Using international indicators to compare equity policies, (pp. 65-92). Dordrecht: Kluwer.

Dicker, G. (2002). Organización y perspectivas de la educación inicial en Iberoamérica: principales 
tendencias. Recuperado de: http://www.oei.es/linea3/diker.pdf

Grupo Asesor Técnico-TAG. (2015). Indicadores temáticos propuestos para el marco de acción de la agendaeducativa2030.Recuperadode:http://unesdoc.unesco.org/images/0023/002332/233247S. pdf

Grupo Consultivo de Atención y Desarrollo de la Primera Infancia. (2016). Informe mundial sobre equidad y la primera infancia. Leiden: Consultative Group on Early Childhood Care and Development (CGECCD).

Hallak, J. (2001). Globalización, derechos humanos y educación. París: Instituto Internacional de Planeamiento de la Educación, Unesco.

Heckman, J. (2006, enero 10). Investing in disadvantaged young children is an economically efficient policy. Documento presentado al Foro del Committee for Economic Development/PEW Charitable Trusts/PNC Financial Services Group forum sobre «Argumentos económicos para efectuar inversiones en la enseñanza preescolar», Nueva York.

Hutmacher, W. (2001). In pursuit of equity in education: Using international indicators to compare equity policies. Dordrecht: Kluwer.

Kernan, M. (2012). Parental involvement in early learning: A review of research, policy and good practice. La Haya: Bernard van Leer Foundation.

Kochen, G. (2013). Aportes conceptuales y experiencias relevantes sobre educación en la primera infancia. Buenos Aires: Instituto Internacional de Planeamiento de la Educación, Unesco.

López, N. (2009). Los temas de la agenda sobre gobierno y dirección de los sistemas educativos en América Latina. En A. Marchesi, J. C. Tedesco, \& C. Coll (Coords.) Calidad, equidad y reformas en la enseñanza, (pp. 35-45). Madrid: OEI.

Marshall, T. H. (1961). Social selection in the Welfare State. En H. Halsey, J. Floud, \& C. A. Anderson (Eds.) Education, economy and society, (pp. 148-164). New York: Free Press.

Mialaret, G. (1976). World survey of pre-school education. París: Unesco.

Montgomery, H., Burr, R., \& Woodhead, M. (2003). Changing childhoods: Local and global. Chichester: Wiley, Open University.

Moreno-Herrera, L. (2005). Equity in education across borders: What are we investigating and discussing? En L. Moreno-Herrera, G. Jones, \& J. Rantala (Eds.) Enacting equity in education, (pp. 13-34). Helsinki: Research Centre for Social Studies on Education, University of Helsinki.

Muñoz, V. (2013). Derechos desde el principio: atención y educación en la primera infancia. Recuperado de: http://www.campaignforeducation.org (14/11/2016).

Myers, R. (2009). El derecho a la educación: calidad y equidad en educación preescolar. Recuperado de: http://www.acude.org.mx/biblioteca/calidad/el-derecho-a-la-educacion-calidad-y-equidad. pdf

Oficina Regional de Educación para América Latina y el Caribe-Orealc. (2013). Education agenda post 2015 Latin America and the Caribbean. Ciudad de México: Unesco, Santiago Office Regional Bureau of Education for Latin America and the Caribbean.

Open Working Group.(2014). Sustainabledevelopment. Recuperadode:http://sustainabledevelopment. un.org/content/documents/45180utcome\%20SDGs.pdf

Organización para la Cooperación y el Desarrollo Económico. (2013). Pisa 2012 results: Excellence through equity: Giving every student the chance to succeed. Recuperado de: http://dx.doi. org/10.1787/9789264201132-en

Organización de Estados Iberoamericanos. (2000). Panorama y perspectivas de la educación inicial en Iberoamérica. X Conferencia Iberoamericana de Educación, OEI, Ciudad de Panamá, Panamá.

Organización de Estados Iberoamericanos. (2010). 2021, metas educativas. La educación que queremos la generación de los bicentenarios. Recuperado de: http://www.oei.es/acercade/ metas 2021

Organización de Estados Iberoamericanos. (2015). Plan de cooperación para el fortalecimiento y extensión de la educación inicial en Iberoamérica. Recuperado de: http://www.oei.es/linea3/ 
plan_cooperacion.htm.

Penn, H. (2008). Early childhood education and care in Southern Africa. Londres: CfBT, Education Trust Reading.

Pérez-Escamilla, R. P., Rizzoli-Córdoba, A., Alonso-Cuevas, A., \& Reyes-Morales, H. (2017). Avances en el desarrollo infantil temprano: desde neuronas hasta programas a gran escala. Boletín Médico del Hospital Infantil de México, 74(2), 86-97. doi: https://doi.org/10.1016/j.bmhimx.2017.01.007

Ramírez, N. (2011). Primera infancia: una agenda pendiente de derechos. En J. Palacios, \& E. Castañeda (Eds.) La primera infancia (0-6 años) y su futuro, (pp. 63-71). Madrid: OEI, Fundación Santillana.

Rosemberg, F. (1999). Expansión de la educación infantil y procesos de exclusión. Cuadernos de Pesquisa, (107), 7-40.

Sen, A. (1992). Inequality re-examined. Oxford: University Press.

Sen, A. (2004). Nuevo Examen de la desigualdad. Madrid: Alianza Editorial.

Sistema de Información de Tendencias Educativas en América Latina-Siteal. (2008). Informe sobre las tendencias sociales y educativas en América Latina 2007. Madrid: Iipe, Unesco, OEI.

Smidt, S. (2010). Key issues in early years education. Londres: Routledge.

Sosinsky, L., Lord, H., \& Zigler, E. (2007). For-profit/non-profit differences in center-based child care quality: Results from the National Institute of Child Health and Human Development Study of Early Child Care and Youth Development. Journal of Applied Developmental Psychology, 28(5), 390-410. doi:10.1016/j.appdev.2007.06.003

Tedesco, J. C. (2009). Los temas de la agenda sobre gobierno y dirección de los sistemas educativos en América Latina. En A. Marchesi, J. C. Tedesco, \& C. Coll (Coords.) Calidad, equidad y reformas en la enseñanza, (pp. 77-86). Madrid: OEI.

Tomasevski, K. (2006). Human rights obligations in education: The 4-A scheme. Nijmegen: Wolf Legal.

Umayahara, M. (2004). En búsqueda de la equidad y calidad de la educación de la primera infancia en América Latina. Revista Latinoamericana de Ciencias Sociales, Niñez y Juventud, 2(2), 21-49.

Unesco. (2004). Informe de seguimiento de la EPT en el mundo 2005. Educación para todos-El imperativo de la calidad. París: Unesco.

Unesco. (2007). Informe de seguimiento de la EPT en el mundo 2007: bases sólidas. La atención y educación de la primera infancia. París: Unesco.

Unesco. (2010, septiembre 27-29). Moscow framework for action and cooperation: Harnessing the wealth of nations. World Conference on Early Childhood Care and Education (Ecce), Moscow, Russia. Recuperado de: http://unesdoc.unesco.org/images/0018/001898/189882e.pdf

Unesco. (2013). Recomendaciones agenda educativa post 2015 en América Latina y el Caribe. Recuperado de:

http://www.unesco.org/new/fileadmin/MULTIMEDIA/FIELD/Santiago/pdf/recomendacionesagenda-educativa-post-2015.pdf

Unesco. (2014 octubre 30-31). Educación para Todos (EPT) en América Latina y el Caribe: balance y desafíos post-2015. Declaración de Lima, Lima, Perú. Recuperado de: http://www.unesco.org/ new/fileadmin/MULTIMEDIA/FIELD/Quito/pdf/Declaracion_lima.pdf

Unesco. (2017). Informe de seguimiento de la educación en el mundo. París: Unesco.

Unesco, Unicef, \& World Bank. (2015). Educación 2030. Declaración de Ichneon y marco de acción para la realización del objetivo de desarrollo sostenible. Recuperado de: http://unesdoc.unesco. org/images/0024/002456/245656s.pdf

Vargas-Baron, E. \& Schipper, J. (2012). Review of policy and planning indicators in early childhood. París: Rise Institute.

Vincent, C. (2003). Social justice, education and identity. London: Routledge Falmer Press.

Woodhead, M., \& Moss, P. (Eds.) (2007). Early childhood and primary education: Transitions in the 
lives of young children. Milton Keynes: The Open University.

Young, M. E. (1996). Desarrollo del niño en la primera infancia: una inversión en el futuro. Washington, D. C.: Banco Mundial.

Young, M. E., \& Fujimoto, G. (2003). Desarrollo infantil temprano: lecciones de los programas no formales. Revista Latinoamericana de Ciencias Sociales, Niñez y Juventud, 1(1), 1-25. Recuperado de: http://www.oei.es/inicial/articulos/desarrollo_infantil_temprano.pdf 